\title{
THE EFFECTS OF SORET AND DUFOUR ON A STEADY MHD FREE CONVECTION FLOW OVER AN INCLINED POROUS PLATE WITH VISCOUS DISSIPATION
}

\author{
O. A. Ajala ${ }^{1}$, I. O. Onikola ${ }^{2}$ \\ 1 Department of Pure and Applied Mathematics, LadokeAkintola University of Technology \\ Ogbomoso, Oyo Nigeria. \\ oaajala@lautech.edu.ng \\ 2 Department of Mathematics and Statistics, Federal Polytechnic Offa, Kwara Nigeria. \\ yemobenson2k@gmail.com
}

\section{ABSTRACT}

This paper deals with the effects of Soretand Dufouron a steady MHD free convection flow over an inclined porous plate with viscous dissipation. The models were non-dimensionalisedand the following parameters arise in the flow: Soret number Sr, Dufour number Du, Prandtl number $\mathrm{Pr}$, magnetic parameter M, Schmidtl number Sc, radiation parameter R, permeability of porous medium K, thermal Grashof number Gr, solutalGrashof number Gm, Eckert number Ec and inclination angle $\alpha$. The influences of these parameters which control the flow were analysedand given a place in this work. The dimensionless governing equations of flow field are solved numerically by collocation weighted residual method. The effects of various parameters on the velocity, temperature and concentration profiles are discussed and displayed graphically

Keywords:Dufour effect, Soret effect, Steady, Viscous Dissipation Heat, MHD, Porous mediumand Collocation weighted residual method

\section{Council for Innovative Research}

\author{
Peer Review Research Publishing System
}

\section{Journal: JOURNAL OF ADVANCES IN MATHEMATICS}

Vol.11, No.9

www.cirjam.com, editorjam@gmail.com 


\section{NOMENCLATURE}

$u^{*} \quad$ Velocity component along $x^{*}$ - direction

$v^{*} \quad$ Velocity component along $y^{*}$ - direction

g Acceleration due to gravity

$\beta \quad$ Volumetric coefficient of thermal expansion

$\beta^{\prime} \quad$ Coefficient of volume expansion for mass transfer

v Kinematic viscosity

$\rho \quad$ Fluid density

$B_{0} \quad$ Magnetic induction

k Thermal conductivity of the fluid

$K^{*} \quad$ Permeability of porous medium

$\sigma \quad$ Electrical conductivity of the fluid

$T^{*} \quad$ Dimensional temperature

$T_{\infty}^{*} \quad$ Temperature of free stream

$C^{*} \quad$ Dimensional concentration

$C_{\infty}^{*} \quad$ Concentration of free stream

$D_{m} \quad$ Chemical molecular diffusivity

$C_{p} \quad$ Specific heat at constant pressure

$K_{T} \quad$ Thermal diffusion rate

$q_{r} \quad$ Radiative heat flux in y direction

\section{INTRODUCTION}

The study of Soret and Dufour effect on MHD flows has attracted considerable attention in the last several decades and is now considered to be of great significant field of study in the general areas of fluid dynamics and heat transfer. There are many relevant applications of this study, these are heat transfer connected with heat recovery from geothermal systems particularly in the field of large storage systems of agricultural produce, heat transfer connected with storage of nuclear waste, exothermic reaction, heat removal from nuclear fuel debris, the heating of rooms and buildings by the use of radiators, control of pollutant spread in groundwater, solar power collectors, radio propagation through the ionosphere, porous material regenerative heat exchangers etc. The study of heat generation in moving fluids is relevant as it changes the temperature distribution and the particle deposition measure especially in nuclear reactor cores, fire and combustion modeling. Whenever heat and mass transfer occur concurrently in a moving fluid, the connection between the fluxes and the driving potentials may be of a more complex type. An energy flux can be created by temperature gradients and by concentration gradients. The energy flux generated by a concentration gradient is called the Dufour or diffusion-thermo effect while the energy fluxgenerated by temperature gradient represents the Soret or thermal-diffusion effect. These effects are very important when the temperature and concentration gradients are very high.

Anghelet al. (2000) studied the Dufour and Soret effects on free convection boundary layer over a vertical surface embedded in a porous medium. Raptiset al. (2003) studied the effect of radiation on two dimensional steady MHD optically thin gray gas flow along an infinite vertical plate in presence of induced magneticfield. Ghaly (2002) investigated the effect of radiation on heat and mass transfer over stretching sheet in the presence of a magnetic field. Lavanya andLeela(2014) Dufour and soret effects on steady MHD free convective flow past a vertical porous plate embedded in a porous medium with chemical reaction, radiation heat generation and viscous dissipation. Chamkha and Ben-Nakhi (2008) studied MHD mixed convection radiation interaction along a permeable surface immersed in a porous medium inthe presence of Soret and Dufour'seffects. Many researchers have investigated Dufour and Soret effects on free convective heat and mass transfer flow in a porous medium, some of them are El-Aziz (2008), Alam and Ahammad (2011), Alam and Rahman (2005), Mansour et al. (2008), Sreedharet al. (2013), Afify (2009). Shivaiah and Anand (2011) studied the effects of soretdufour and thermal radiation on MHD free convection flow past an infinite vertical porous plate in the presence of chemical reaction. Sarada and Shanker (2013) analysed the effects of soret and dufour on MHD flowalong a vertical porous plate in the presence of suction or injection.

The aim of this paper is to study the effects of Soretand Dufouron a steady MHD free convection flow over an inclined porous plate with viscous dissipation. The set of governing equations and boundary equation of the problem are transformed into a set of nonlinear ordinary differential equation and solved numerically by collocation weighted residual method.The effects of various parameters on the velocity, temperature and concentration profiles are discussed and displayed graphically. 


\section{MATHEMATICAL ANALYSIS}

A steady-state two-dimensional heat and mass transferflow of an incompressible and electrical conducting viscous fluid along an infinite incline porous plate embedded in a porous medium with viscous dissipation is considered. The plate is inclined at angle $\alpha$ to the vertical with the $x^{*}$ - axis taken along the plate and $y^{*}$ - axis taken normal to it. It is assumed that the radiation heat flux in $x^{*}$ - direction is negligible in comparison to $y^{*}$ - direction. Initially the plate and the fluid are at the same temperature $T_{\infty}^{*}$ in a stationary condition with concentration level $C_{\infty}^{*}$ at all points. The plate starts moving impulsively in its own plane with velocity $\mathrm{u}_{0}$, its temperature is raised to $T_{w}$ and the concentration level at the plate is raised to $C_{w}$. A transverse magnetic field of uniform strength $B_{0}$ is assumed normal to the direction of flow. The transversely applied magnetic field and magnetic Reynolds number are very small and hence induced magnetic field is negligible, Cowling (1957).

As a result of infinite length in $x^{*}$ - direction, the flow variables are functions of $y^{*}$ only. On this physical grounds of the present problem and under the usual Boussinesq approximation, governing equations for this steady problem are given by

Continuity equation:

$\frac{d v^{*}}{d y^{*}}=0 \Rightarrow v^{*}=-v_{0}$

Momentum equation:

$v^{*} \frac{d u^{*}}{d y^{*}}=v \frac{d^{2} u^{*}}{d y^{*}}+g \beta\left(T^{*}-T_{\infty}^{*}\right) \cos (\propto \propto)+g \beta^{\prime}\left(C^{*}-C_{\infty}^{*}\right) \cos ((\propto))-\frac{V U^{*}}{K^{*}}-\frac{\sigma B_{0}^{2} u^{*}}{\rho}$

Energy equation:

$$
\rho C_{p} v^{*} \frac{d T^{*}}{d y^{*}}=k \frac{d^{2} T^{*}}{d y^{*}}-\frac{d q_{r}}{d y^{*}}+\frac{\rho D_{m} K_{T}}{C_{s}} \frac{d^{2} C^{*}}{d y^{* 2}}+\mu\left(\frac{d u^{*}}{d y^{*}}\right)^{2}
$$

Concentration equation:

$$
v^{*} \frac{d C^{*}}{d y^{*}}=D_{m} \frac{d^{2} C^{*}}{d y^{*}}+\frac{D_{m} K_{T}}{T_{m}} \frac{d^{2} T^{*}}{d y^{*}}
$$

where $u^{*}$ is the velocity component along $x^{*}$ - direction, $v^{*}$ is the velocity component along $y^{*}$ - direction, $\mathrm{g}$ is the acceleration due to gravity, $\beta$ is the volumetric coefficient of thermal expansion, $\beta^{\prime}$ is the coefficient of volume expansion for mass transfer, $T^{*}$ is the dimensional temperature, $T_{\infty}^{*}$ is temperature of free stream, $C^{*}$ is the dimensional concentration, $C_{\infty}^{*}$ is concentration of free stream, $\mathrm{v}$ is the kinematic viscosity, $\rho$ is the fluid density, $B_{0}$ is the magnetic induction, $\mathrm{k}$ is the thermal conductivity of the fluid, $K^{*}$ is the permeability of porous medium, $D_{m}$ is the chemical molecular diffusivity, $\sigma$ is the electrical conductivity of the fluid, $C_{p}$ is the specific heat at constant pressure, $K_{T}$ is the thermal diffusion rate, $q_{r}$ is radiative heat flux in y direction.

The initial and boundary conditions for the model are given by

$u^{*}=0, T^{*}=T_{\infty}^{*}, C^{*}=C_{\infty}^{*} \quad \forall y^{*}$

$u^{*}=u_{0}, v^{*}=-v_{0}, T^{*}=T_{\infty}^{*}+\left(T_{w}^{*}-T_{\infty}^{*}\right) e^{o}$

$C^{*}=C_{\infty}^{*}+\left(C_{w}^{*}-C_{\infty}^{*}\right) e^{o}$ at $y^{*}=0$

$u^{*}=0, T^{*} \rightarrow T_{\infty}^{*}, C^{*} \rightarrow C_{\infty}^{*}, y^{*} \rightarrow \infty$

By using Roseland approximation, the radiative heat flux term is given by

$q_{r}=-\frac{4 \sigma}{3 k_{1}} \frac{d T^{* 4}}{d y^{*}}$

where $k_{1}$ and $\sigma$ are mean absorption coefficient and Stefan Boltzmann constant respectively. Assuming the temperature difference within the flow are copiously small such that $T^{* 4}$ can be expressed as a linear function of the temperature. It is accomplished by expanding in a Taylor series about $T_{\infty}^{* 4}$ and neglecting the higher order terms, hence

$T^{* 4} \cong 4 T_{\infty}^{* 3} T^{*}-3 T_{\infty}^{* 4}$

Putting (6) and 7 into (3) gives

$\rho C_{p} v^{*} \frac{d T^{*}}{d y^{*}}=k \frac{d^{2} T^{*}}{d y^{* 2}}+\frac{16 \sigma T_{\infty}^{* 3} d^{2} T^{*}}{3 k_{1} d y^{* 2}}+\frac{\rho D_{m} K_{T}}{C_{s}} \frac{d^{2} C^{*}}{d y^{*^{2}}}+\mu\left(\frac{d u^{*}}{d y^{*}}\right)^{2}$

In order to write the governing equations and the boundary condition in dimensionless form, the following non-dimensional quantities are introduced.

$u=\frac{u^{*}}{u_{0}}, y=\frac{y^{*} V_{0}}{v}, \theta=\frac{T^{*}-T_{\infty}^{*}}{T_{\omega}^{*}-T_{\infty}^{*}}, C=\frac{C^{*}-C_{\infty}^{*}}{C_{w}^{*}-C_{\infty}^{*}}, U_{P}=\frac{U_{P}^{\prime}}{V_{0}}, G_{r}=\frac{g \beta v\left(T_{\omega}^{*}-T_{\infty}^{*}\right)}{U_{0} V_{0}^{2}}$,

$G_{m}=\frac{g \beta^{\prime} v\left(C_{\omega}^{*}-C_{\infty}^{*}\right)}{U_{0} V_{0}^{2}}, K=\frac{K^{*} V_{0}^{2}}{V^{2}} \quad P_{r}=\frac{v \rho C_{P}}{\kappa}, \quad P_{r}=\frac{\square C_{P}}{\kappa} S_{C}=\frac{v}{D_{m}}, M=\frac{\sigma B_{0}^{2} v}{\rho V_{0}^{2}}$

$D u=\frac{D_{m} K_{T}\left(C_{\omega}^{*}-C_{\infty}^{*}\right)}{c_{s} c_{p} v\left(T_{\omega}^{*}-T_{\infty}^{*}\right)}, S r=\frac{D_{m} K_{T}\left(T_{\omega}^{*}-T_{\infty}^{*}\right)}{T_{m} v\left(C_{\omega}^{*}-C_{\infty}^{*}\right)}, R=\frac{4 \sigma T_{\infty}^{* 3}}{k_{1} k}, E_{C}=\frac{u_{0}^{2}}{C_{P}\left(T_{\omega}^{*}-T_{\infty}^{*}\right)}$, 
Hence, using the above non-dimensional quantities, the equations (2), (4) and (8) in the non-dimensional form can be written as

$\frac{-d u}{d y}=\frac{d^{2} u}{d y^{2}}+\theta G_{r} \cos (\propto)+C G_{m} \cos (\propto)-\left(M+\frac{1}{K}\right) u$

$-\frac{d \theta}{d y}=\frac{1}{P_{r}}\left(1+\frac{4 R}{3}\right) \frac{d^{2} \theta}{d y^{2}}+D u \frac{d^{2} C}{d y^{2}}+E_{C}\left(\frac{d u}{d y}\right)^{2}$

$-\frac{d C}{d y}=\frac{1}{S_{C}} \frac{d^{2} C}{d y^{2}}+\operatorname{Sr} \frac{d^{2} \theta}{d y^{2}}$

The corresponding initial and boundary conditions are

$$
\begin{aligned}
& u=0, \theta=0, C=0 \quad \forall y \\
& u=1, \theta=1, C=1 \text { at } y=0 \\
& u=0, \theta \rightarrow 0, C \rightarrow 0, y \rightarrow \infty
\end{aligned}
$$

\section{SOLUTION OF THE PROBLEM}

The system of coupled non-linear ordinary differential equation (10)-(12) together with the boundary conditions (13) are solved numerically by using the method of collocation weighted residual. First of all, we shall assume trial solutions for equations (10) - (12) as given below

$$
\begin{aligned}
& u(y)=a_{0}+a_{1} y+a_{2} y^{2}+a_{3} y^{3}+a_{4} y^{4}+a_{5} y^{5}+a_{6} y^{6}+a_{7} y^{7}+a_{8} y^{8} \\
& \theta(y)=b_{0}+b_{1} y+b_{2} y^{2}+b_{3} y^{3}+b_{4} y^{4}+b_{5} y^{5}+b_{6} y^{6}+b_{7} y^{7}+b_{8} y^{8} \\
& C(y)=c_{0}+c_{1} y+c_{2} y^{2}+c_{3} y^{3}+c_{4} y^{4}+c_{5} y^{5}+c_{6} y^{6}+c_{7} y^{7}+c_{8} y^{8}
\end{aligned}
$$

differentiating (14), (15) and (16) and substituting into (10), (11) and (12) respectively, gives

$$
\begin{aligned}
u(y) & =56 a_{8} y^{6}+42 a_{7} y^{5}+30 a_{6} y^{4}+20 a_{5} y^{3}+12 a_{4} y^{2}+6 a_{3} y+2 a_{2}+a_{1}+2 a_{2} y \\
& +3 a_{3} y^{2}+4 a_{4} y^{3}+5 a_{5} y^{4}+6 a_{6} y^{5}+7 a_{7} y^{6}+8 a_{8} y^{7}+G r \cos (\alpha)\left(b_{0}+b_{1} y+b_{2} y^{2}\right. \\
& \left.+b_{3} y^{3}+b_{4} y^{4}+b_{5} y^{5}+b_{6} y^{6}+b_{7} y^{7}+b_{8} y^{8}\right)+G m \cos (\alpha)\left(c_{0}+c_{1} y+c_{2} y^{2}+c_{3} y^{3}\right. \\
& \left.+c_{4} y^{4}+c_{5} y^{5}+c_{6} y^{6}+c_{7} y^{7}+c_{8} y^{8}\right)-\left(M+\frac{1}{k}\right)\left(a_{0}+a_{1} y+a_{2} y^{2}+a_{3} y^{3}+a_{4} y^{4}\right. \\
& \left.+a_{5} y^{5}+a_{6} y^{6}+a_{7} y^{7}+a_{8} y^{8}\right)
\end{aligned}
$$$$
\theta(y)=\frac{\left(56 y^{6} b_{8}+42 y^{5} b_{7}+30 y^{4} b_{6}+20 y^{3} b_{5}+12 y^{2} b_{4}+6 y b_{3}+2 b_{2}\right)\left(1+\frac{4}{3} R\right)}{P r}
$$$$
+b_{1}+2 b_{2} y+3 b_{3} y^{2}+4 b_{4} y^{3}+5 b_{5} y^{4}+6 b_{6} y^{5}+7 b_{7} y^{6}+8 b_{8} y^{7}+D u\left(56 y^{6} c_{8}\right.
$$$$
\left.+42 y^{5} c_{7}+30 y^{4} c_{6}+20 y^{3} c_{5}+12 y^{2} c_{4}+6 y c_{3}+2 c_{2}\right)+E c\left(56 y^{6} a_{8}+42 y^{5} a_{7}\right.
$$$$
\left.+30 y^{4} a_{6}+20 y^{3} a_{5}+12 y^{2} a_{4}+6 y a_{3}+2 a_{2}\right)^{2}
$$$$
C(y)=\frac{56 y^{6} c_{8}+42 y^{5} c_{7}+30 y^{4} c_{6}+20 y^{3} c_{5}+12 y^{2} c_{4}+6 y c_{3}+2 c_{2}}{S c}+c_{1}+2 c_{2} y
$$$$
+3 c_{3} y^{2}+4 c_{4} y^{3}+5 c_{5} y^{4}+6 c_{6} y^{5}+7 c_{7} y^{6}+8 c_{8} y^{7}+\operatorname{Sr}\left(56 y^{6} b_{8}+42 y^{5} b_{7}\right.
$$$$
\left.+30 y^{4} b_{6}+20 y^{3} b_{5}+12 y^{2} b_{4}+6 y b_{3}+2 b_{2}\right)
$$

Choosing some points within the boundary conditions and collocating each of the equations (17) - (19) at these points yields system of equations which are solved concurrently with their respective boundary conditions.

\section{RESULTS AND DISCUSSION}


From the numerical computations, non-dimensional velocity, temperature and concentration profiles as well as the skinfriction coefficient, Nusselt number and Sherwood number are found for different values of the various physical parameters appearing in the problem. The value of Prandtl number Pris taken to be 0.71 which correspondsto air and the value of Schmidt number Scis chosen to be 0.66 which represents water vapour. However the values ofSoret number Sr, Dufour number Du, Prandtl number Pr, magnetic parameter M, Schmidtl number Sc, radiation parameter R, permeability of porous medium K, thermal Grashof number $\mathrm{Gr}$, solutalGrashof number $\mathrm{Gm}$, Eckert number Ec and inclination angle $\propto$ are chosen arbitrarily.

Figure 1 illustrates the velocity profile for various values of Soret number Sr. The figure shows that increasing Soret number Sr, first velocity decreases and later increases. Figure 2 shows that, an increase in Soret number gives rise to temperature. In figure 3, we observe that concentration decreases at first and later increases as Soret number $\mathrm{Sr}$ increases. It is clearly seen that there is no change in the velocity profile of the Dufour number as shown in figure 4 . Figure 5 illustrates the effect of Dufour number Du on the temperature profile. It is seen that increase in Du leads to increase in temperature of the fluid. The effect of Dufour number Du on the concentration profile is presented in figure 6 . From this figure, we observe that concentration decreases when Du increases. The influence of the Eckert number Ec on velocity profile, temperature profile and concentration profile is illustrated in figures 7-9. It is analysed in these figures that as Ec increases the velocity and temperature of the fluid increase while that of concentration decreases respectively. From Figures 10 and 11, it is observed that both the velocity and temperature profiles decrease with an increase in radiation parameter while the concentration profile increases first and later decreases in figure 12. Figures $13-15$ illustrate the effect of Schmidt number Sc on the velocity, temperature and concentration. It is noticed in figures 13 and 15 that as the Schmidt number Sc increases, a decreasing trend in both the velocity and concentration fields are noticed while that of temperature decreases in figure 14. Also figures 16 - 18 depict the effect of the Prandtl number Pr on the velocity, temperature and concentration. It is observed that as the Prandtl number increases, both the velocity and concentration profiles decrease first and increase later in figure 16 and 18 while in figure 17, temperature profile increases.

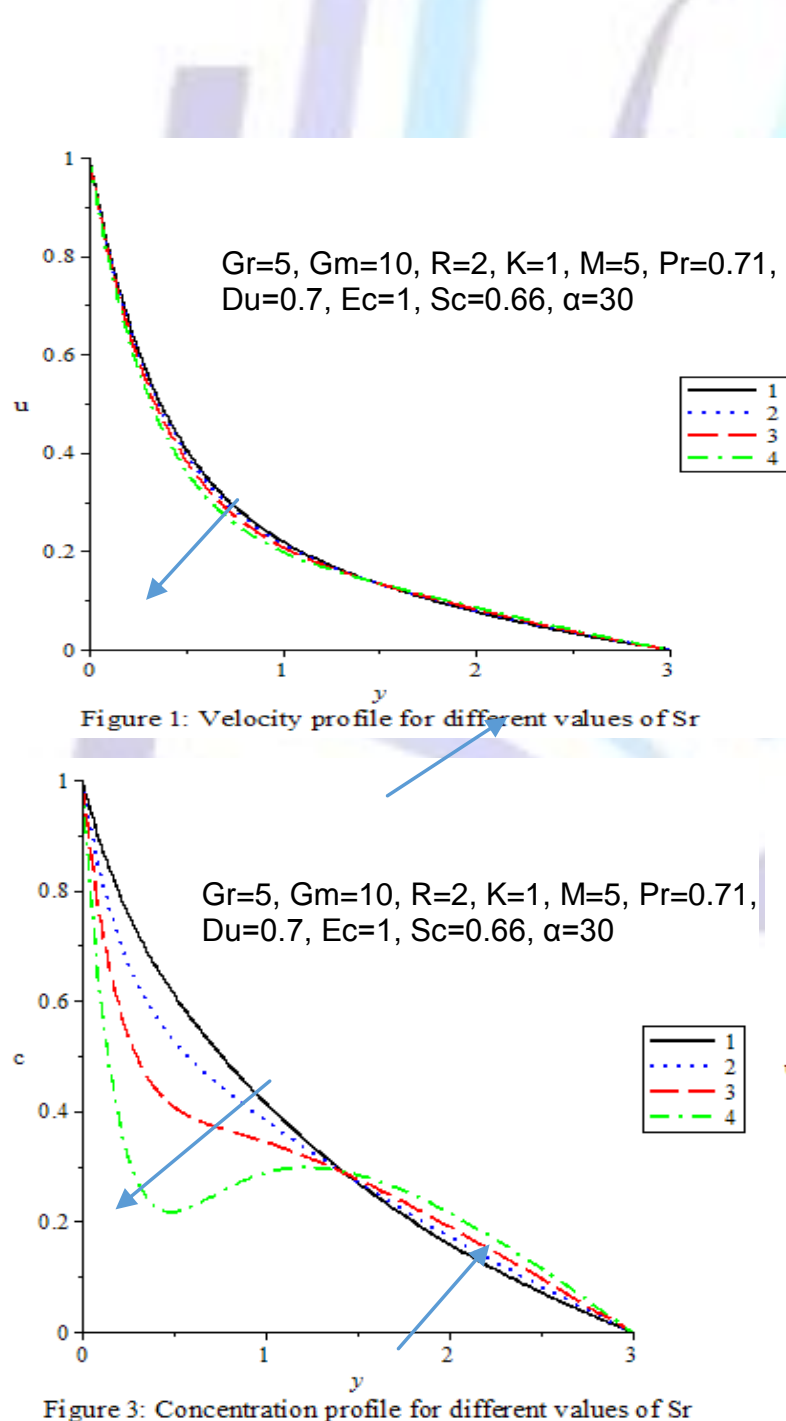

Figure 3: Concentration profile for different values of $\mathrm{Sr}$

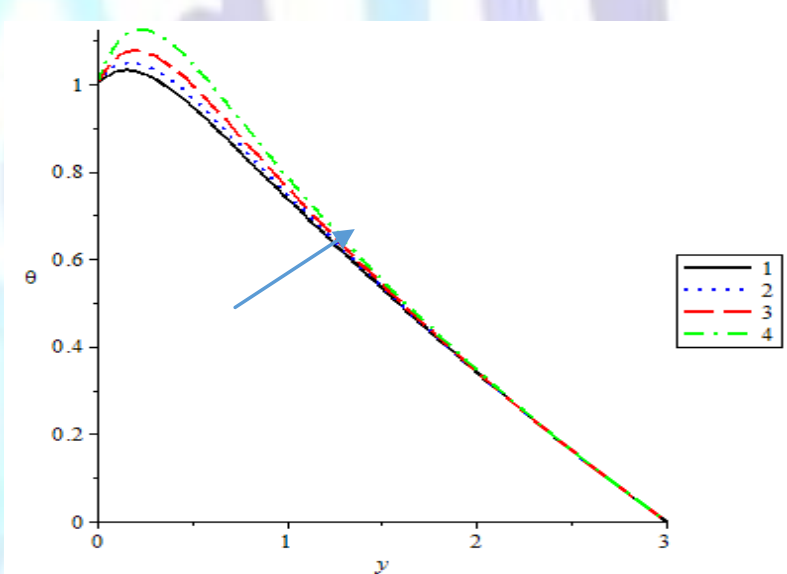

Figure 2: Temperature pro file for different values of $\mathrm{Sr}$

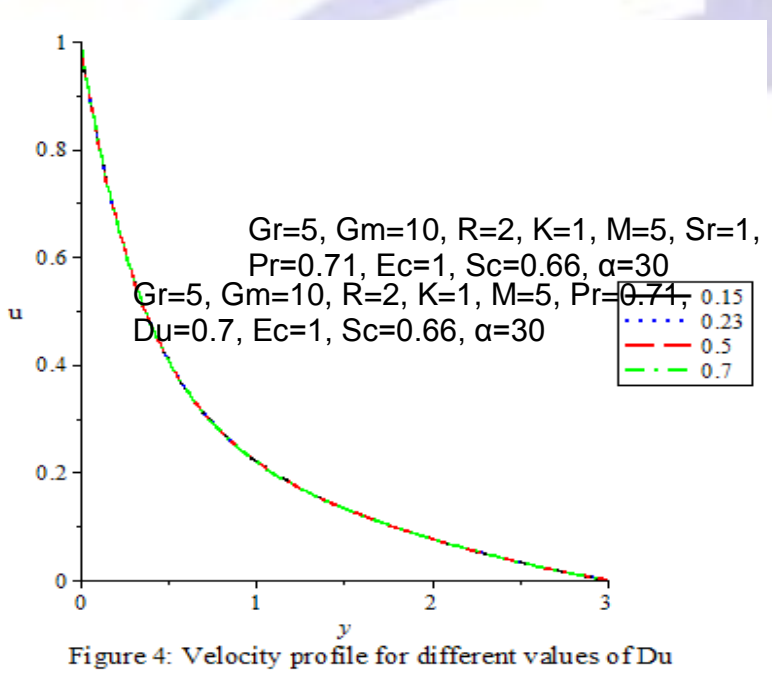

Figure 4: Velocity profile for different values of Du
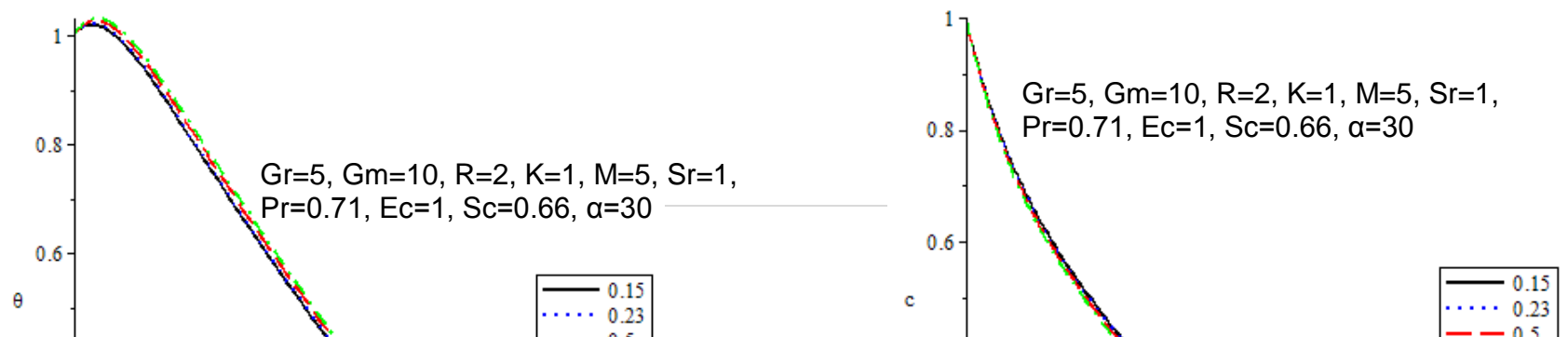


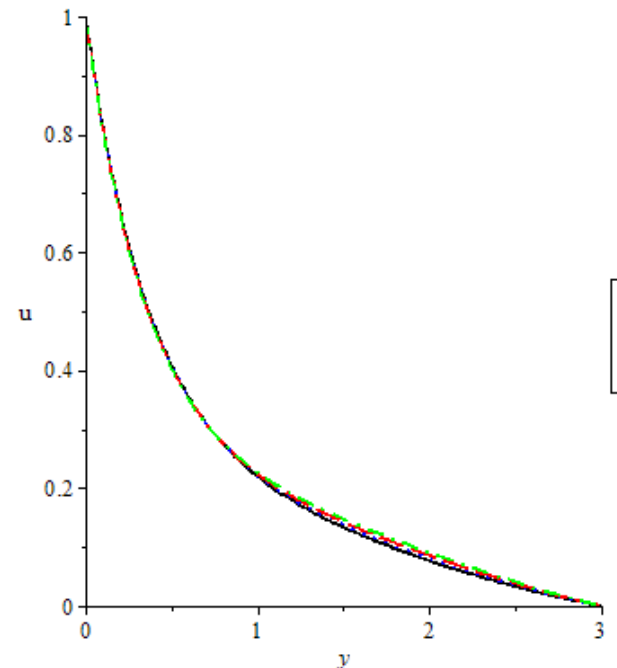

Figure 7: Velocity profile for different values of Ec

$\mathrm{Gr}=5, \mathrm{Gm}=10, \mathrm{Ec}=1, \mathrm{~K}=1, \mathrm{M}=5, \mathrm{Sc}=0.66$ $\mathrm{Du}=0.7, \operatorname{Pr}=0.71, \mathrm{Sr}=1, \alpha=30$

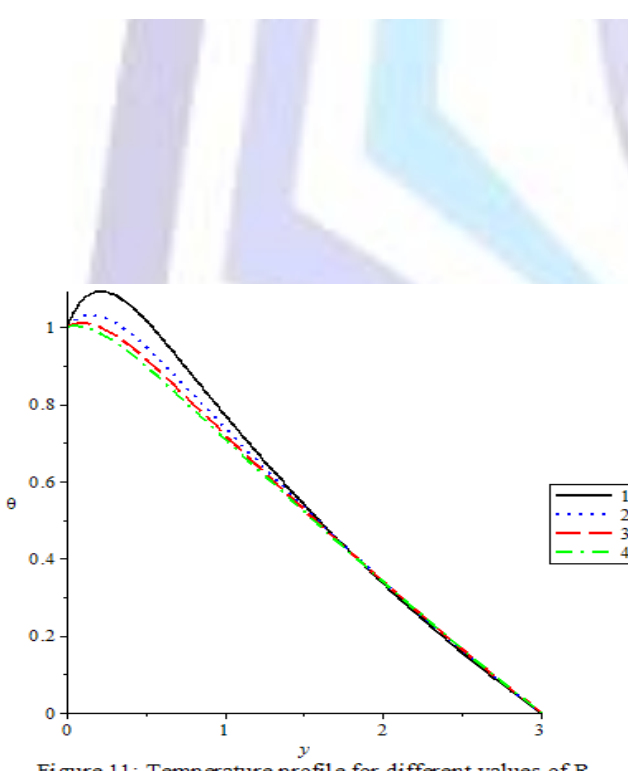

$\mathrm{Gr}=5, \mathrm{Gm}=10, \mathrm{Ec}=1, \mathrm{~K}=1, \mathrm{M}=5, \mathrm{Sc}=0.66$, $\mathrm{Du}=0.7, \mathrm{Pr}=0.71, \mathrm{Sr}=1, \alpha=30$

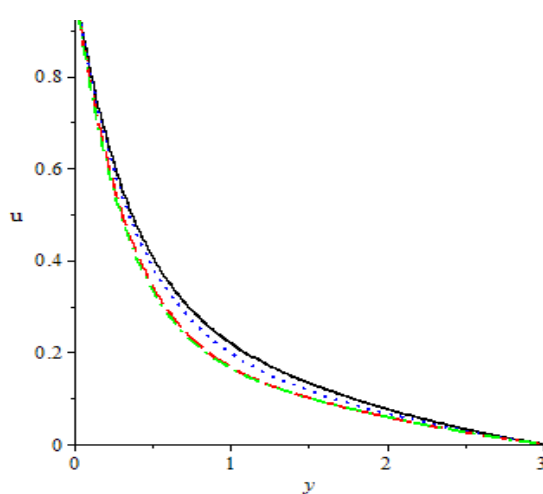

Figure 13: velocity profile for different values of Sc

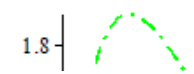

$\mathrm{Gr}=5, \mathrm{Gm}=10, \mathrm{R}=2, \mathrm{~K}=1, \mathrm{M}=5, \mathrm{Sc}=0.66$,

$\mathrm{Du}=0.7, \operatorname{Pr}=0.71, \mathrm{Sr}=1, \alpha=30$

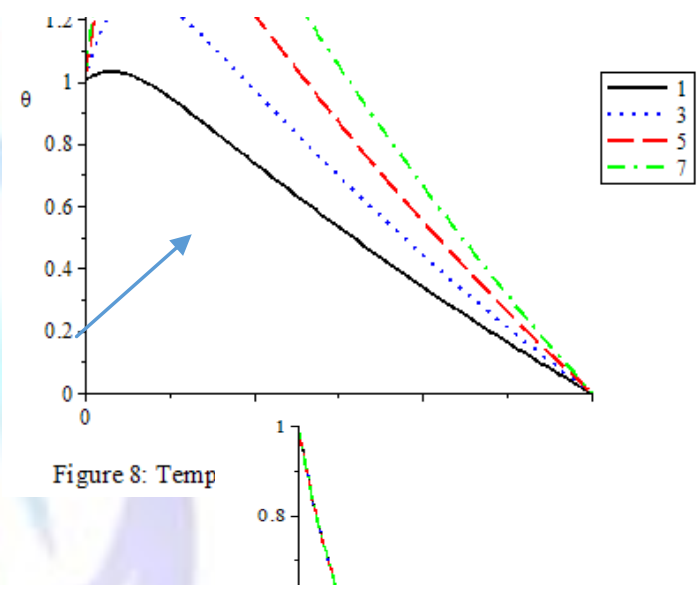

$\mathrm{Gr}=5, \mathrm{Gm}=10, \mathrm{R}=2, \mathrm{~K}=1, \mathrm{M}=5, \mathrm{Sc}=0.66$,

$\mathrm{Du}=0.7, \mathrm{Pr}=0.71, \mathrm{Sr}=1, \alpha=30$
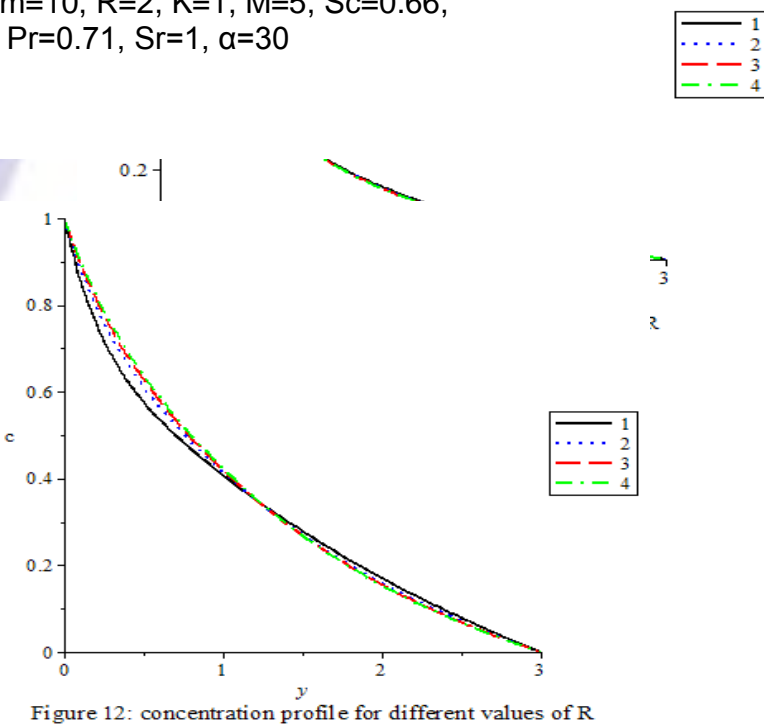

$\mathrm{Gr}=5, \mathrm{Gm}=10, \mathrm{Ec}=1, \mathrm{~K}=1, \mathrm{M}=5, \mathrm{Sc}=0.66$, $\mathrm{Du}=0.7, \operatorname{Pr}=0.71, \mathrm{Sr}=1, \alpha=30$

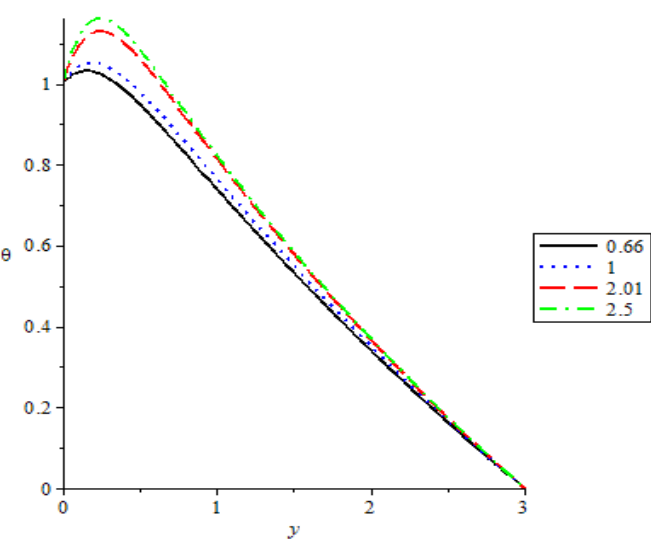

Figure 14: temperature profile for different values of Sc 
$\mathrm{Gr}=5, \mathrm{Gm}=10, \mathrm{Ec}=1, \mathrm{~K}=1, \mathrm{M}=5, \mathrm{R}=2$, $\mathrm{Du}=0.7, \mathrm{Pr}=0.71, \mathrm{Sr}=1, \alpha=30$

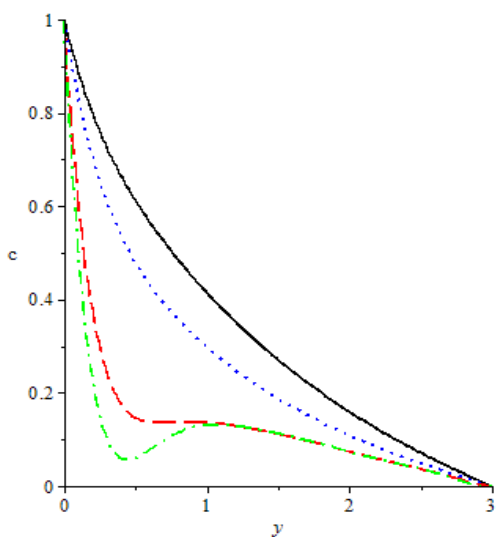

Figure 15: concentration profile for different values of Sc

$\mathrm{Gr}=5, \mathrm{Gm}=10, \mathrm{Ec}=1, \mathrm{~K}=1, \mathrm{M}=5, \mathrm{R}=2$, $\mathrm{Du}=0.7, \mathrm{Pr}=0.71, \mathrm{Sr}=1, \alpha=30$

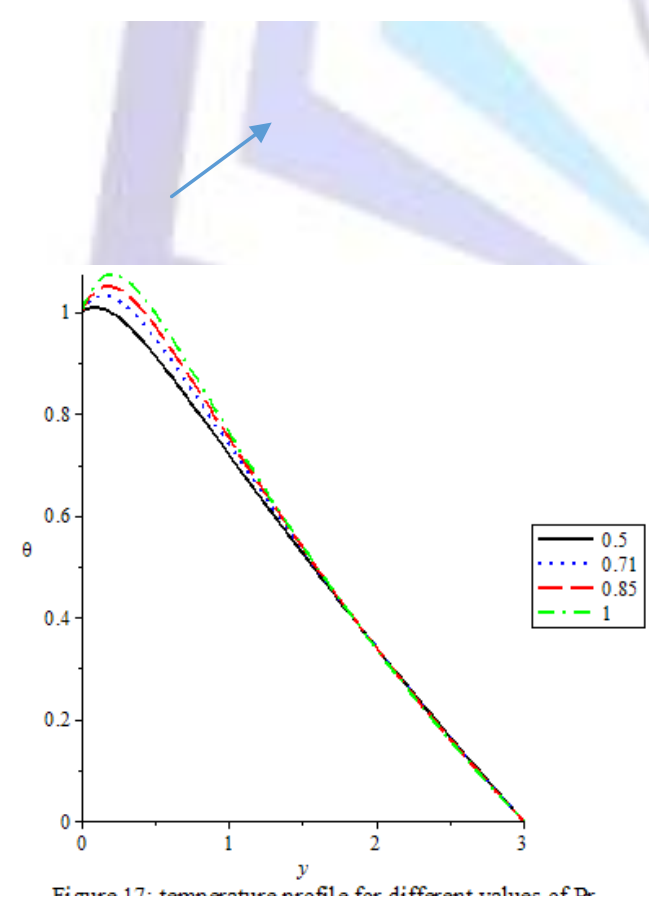

$\mathrm{Gr}=5, \mathrm{Gm}=10, \mathrm{Ec}=1, \mathrm{~K}=1, \mathrm{M}=5, \mathrm{R}=2$, $\mathrm{Du}=0.7, \mathrm{Sc}=0.66, \mathrm{Sr}=1, \alpha=30$
$\mathrm{Gr}=5, \mathrm{Gm}=10, \mathrm{Ec}=1, \mathrm{~K}=1, \mathrm{M}=5, \mathrm{R}=2$, $\mathrm{Du}=0.7, \operatorname{Pr}=0.71, \mathrm{Sr}=1, \alpha=30$

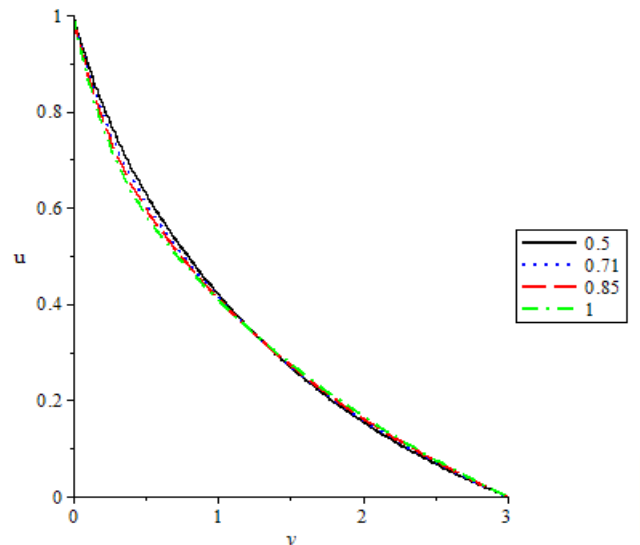

Figure 16: velocity profile for different values of Pr

$\mathrm{Gr}=5, \mathrm{Gm}=10, \mathrm{Ec}=1, \mathrm{~K}=1, \mathrm{M}=5, \mathrm{R}=2$, $\mathrm{Du}=0.7, \mathrm{Sc}=0.66, \mathrm{Sr}=1, \alpha=30$

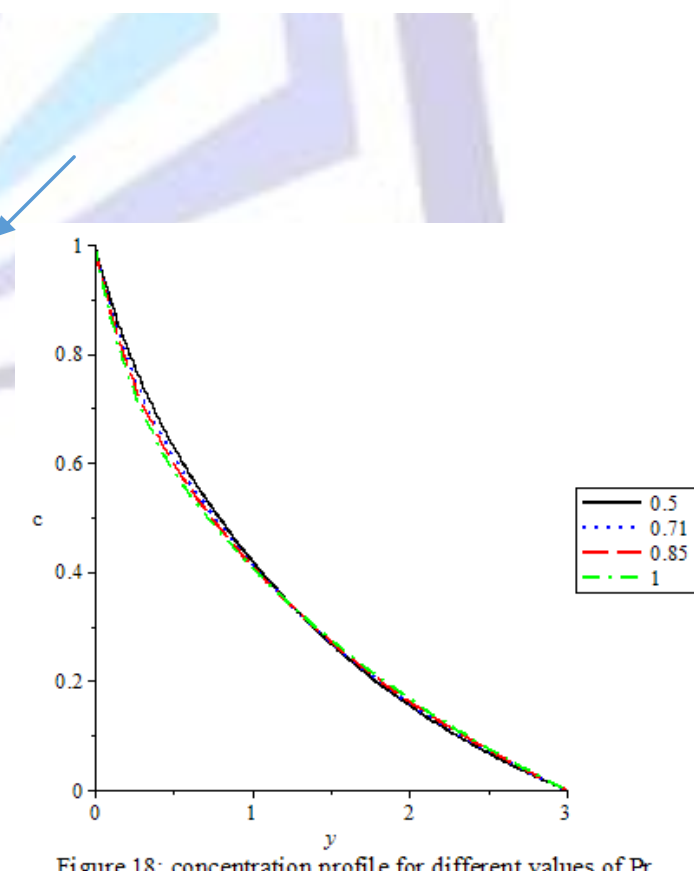

Figure 18: concentration profile for different values of $\mathrm{Pr}$ $\mathrm{Gr}=5, \mathrm{Gm}=10, \mathrm{Ec}=1, \mathrm{~K}=1, \mathrm{M}=5, \mathrm{R}=2$, $\mathrm{Du}=0.7, \mathrm{Sc}=0.66, \mathrm{Sr}=1, \alpha=30$

\section{CONCLUSION}


The main goal for this present work is to study the effects of soret and dufour on a steady MHD free convection flow over an inclined porous plate with viscous dissipation. This was achieved by first formulating the problem under reasonable assumptions. The qualitative properties of the models were studied using mathematical approach. The model is nondimentionalised using the following non-dimentional quantities that govern the flow situation: Soret number Sr, Dufour number Du, Prandtl number Pr, magnetic parameter M, Schmidtl number Sc, radiation parameter R, permeability of porous medium K, thermal Grashof number Gr, solutalGrashof number Gm, Eckert number Ec and inclination angle $\propto$ The resulting coupled non-linear ordinary differential equations are solved numerically by collocation weighted residual method after the method has been tested for efficiency.

In other to have better physical insight of this problem, the results are presented in graphs. Hence from present numerical study we conclude that

(1) The velocity and concentration profiles decrease first and later increase while that of temperature increases as the Soretnumber increases.

(2) An increase in Dufour number leads to increase in temperature of the fluid and a decrease in concentration profile.

(3) Increasing Eckert number enhances both the velocity and temperature distributions in the flow region while concentration profile decreases.

(4) Both velocity and concentration decrease afterward increase when Prandtl number increases while temperature increases.

(5) Both velocity and concentration decrease on increasing Schmidtl number

(6) The temperature profile is observed to increase with an increase Schmidt number

\section{REFERENCES}

[1] Afify A.A. (2009).Commun. Nonlinear Sci. Numer. Simul, no 14 pp 2204-2214.

[2] Alam M.S and Rahman M.M (2005).J. Naval Architecture and Marine Engineering, vol2 no 1 pp 55-65.

[3] Alam M.S. and Ahammad M.U. (2011).Nonlinear Analysis: Modelling and Control, vol 16 no 1 pp 1-16.

[4] Anghel M, Takhar H.S and Pop I M (2000).Stud. Univ. Babes-Bolyai, Math, no 45 pp. 11-21.

[5] Chamkha A.J and Ben-Nakhi A. (2008).Heat Mass Transfer, no 44 pp 845-856.

[6] El-Aziz M.A. (2008).Phys. Lett. A, no 372 pp 263-272.

[7] Ghaly A.Y. (2002).Radiation effect on a certain MHD free convection flow," Chaos, Solution and Fractals, vol. 13(9), pp. 1843-1850.

Lavanya B. and A. LeelaRatnam (2014).Dufour and soret effects on steady MHD free convective flow past a vertical porous plate embedded in a porous medium with chemical reaction, radiation heat generation and viscous dissipation. Advances in Applied Science Research, no 5 pp 1:127-142

Mansour M.A, El-Anssary N.F and Aly A.M(2008).Journal of Chemical Engineering, vol 145 no 2 pp 340-345.

[10] Raptis A., C. Perdikis and A. Leontitsis, (2003). Effect of radiation in an optically thin gray gas flowing past a vertical infinite plate in presence of a magnetic field. Heat and Mass Transfer, vol. 39, pp. 771-773.

[11] Sarada K. and Shanker B. (2013).The effects of soret and dufour on an unsteady MHD free convection flow past a vertical porous plate in the presence of suction or injection. International

[12] Journal of Engineering and Sciencepp2278-4721, vol 2 pp13-25.

[13] Shivaiah S. and Anand J.R. (2011).Effects of Soretdufour and thermal radiation on unsteady MHD free convection flow past an infinite vertical porous plate in the presence of chemical reaction. Int. J. of Appl. Math and Mech. vol7no 13 pp 58-76.

[14] SreedharSarma G, Rama Krishna Prasad and Govardhan K (2013).IOSR Journal of Mathematics, vol 8 no 2 pp 67-87. 